\title{
Research and Design of High Torque Soft Starter Based on Discrete Frequency
}

\author{
Tong Jun ${ }^{1, a}$, Lu Yao ${ }^{1, b}$ and Wang Xia-hui ${ }^{1, c}$ \\ ${ }^{1}$ School of Electrical and Control Engineering, Xi'an University of Science and Technology \\ ,Xi'an 710054, China \\ axkdtongjun@163.com, ${ }^{\mathrm{b}} 383755981 @ q q . c o m,{ }^{\mathrm{c}} 583311943 @ q q . c o m$
}

Keywords: induction motor; soft starter; discrete frequency; equivalent sine wave principle; VVVF

\begin{abstract}
By deeply analyzing discrete frequency theory, this thesis put forward rational theory basis about choosing discrete frequency spectrum and phase position. On the basis of traditional discrete frequency soft starter, this thesis utilize control strategy based on equivalent sine wave principle to promote the original system. By using MATLAB/Simulink tool, we built the discrete frequency simulation platform and acquired torque, rotate speed and simulated wave of output current. We tested the wave on experimental platform and verified that all theory we came up with are correct and rational.
\end{abstract}

\section{Introduction}

In Tesla numerous inventions, the most benefit of the public are the three-phase AC and AC motors. Even three-phase asynchronous motors have many advantages, they still have shortcomings. That is, direct start will produce a great starting current, which is about $4 \sim 7$ times the rated value, even 10 times under overload. How to reduce motor starting current and maintain a high starting torque have attracted the attention of domestic and foreign experts. Using the theory of discrete frequency control, this paper has developed a new type of discrete frequency of high torque soft starter, as in [1], and solves the problem of large motor starting current effectively.

\section{Study on Discrete Frequency Method}

\subsection{Discrete Frequency Principle}

Discrete frequency is controlled by cycle: namely, some half AC voltage waveform are turn-on and some are cut-off, so as to increase the output voltage period to reduce the frequency. Because the changes of period have integer multiple relations, the frequency have the same relationship. The discrete frequency soft starter is the process of switching frequency between them, so the discrete frequency is also known as hierarchical frequency. The discrete frequency softer starter can make the motor acquire the similar effect of VVVF converter, and improve the initial starting torque of three-phase induction motor effectively (e.g. [2],[3]).

Discrete frequency soft starter still uses main circuit topology of traditional electronics soft starter. Its control strategy consider not only the period but also the phase, so it can be easy to think is the superimposition of a phase control and cycle control.

Fig. 1 shows the process of single phase discrete frequency that follow7.14Hz $\rightarrow 8.33 \mathrm{~Hz} \rightarrow 10 \mathrm{~Hz} \rightarrow 12.5 \mathrm{~Hz} \rightarrow 16.7 \mathrm{~Hz} \rightarrow 25 \mathrm{~Hz}$. But three-phase discrete frequency is more complex than single-phase, because it need to consider power phase sequence and etc.

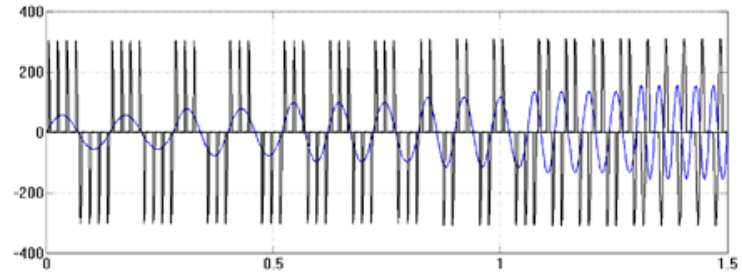

Fig. 1 Waveform of single-phase discrete frequency for $7.14 \rightarrow 8.33 \rightarrow 10 \rightarrow 12.5 \mathrm{~Hz} \rightarrow 16.7 \mathrm{~Hz} \rightarrow 25 \mathrm{~Hz}$ 


\subsection{Selection of Discrete Frequency Phase Sequence and Phase}

Three-phase AC instantaneous value of the voltage can be expressed as

$$
\left\{\begin{array}{l}
u_{A 1}=U_{1} \bullet \sin \left(\omega_{\text {net }} t\right) \\
u_{B 1}=U_{1} \bullet \sin \left(\omega_{\text {net }} t-2 / 3 \pi\right) \\
u_{C 1}=U_{1} \bullet \sin \left(\omega_{\text {net }} t-4 / 3 \pi\right)
\end{array}\right.
$$

After the frequency division, the instantaneous value of the three-phase voltage can be expressed as

$$
\left\{\begin{array}{l}
u_{A v}=U_{1 v} \cdot \sin \left(\omega_{1 v} t-\varphi_{A v}\right) \\
u_{B v}=U_{1 v} \bullet \sin \left(\omega_{1 v} t-\varphi_{B v}\right) \\
u_{C v}=U_{1 v} \cdot \sin \left(\omega_{1 v} t-\varphi_{C v}\right)
\end{array}\right.
$$

where $\omega_{n e t}=v \omega_{1 v}, \varphi_{A v}$ must be a root of the formula $\omega_{\text {net }} t=k \pi(k=0,2,3 \cdots)$, $\operatorname{sos} \sin \left(\omega_{\text {net }} t-\varphi_{A v}\right)=0$. In the same way, we can acquire $\varphi_{B v}, \varphi_{C v}$ as follows

$$
\left\{\begin{array}{l}
\varphi_{A v}=\frac{k \pi}{v} \\
\varphi_{B v}=\frac{2 / 3 \pi+k \pi}{v} \\
\varphi_{C v}=\frac{4 / 3 \pi+k \pi}{v}
\end{array} \quad k=0,1,2, \cdots, 2 v-1\right.
$$

When is equal to $3 \mathrm{k}+1$, three-phase discrete voltage has positive order; When is equal to 3k-1,it has negative order; When is equal to $3 \mathrm{k}$, it only has zero-order component. Meanwhile, for the positive and negative phase order in three-phase discrete voltage, odd frequency can obtain the phase, amplitude and the time axis balance (e.g. [4],[5]).

Selecting the frequency division series should pay more attention to the following points:

First, consider the positive order and odd frequency in three-phase discrete voltage, such as 13 and 7 frequency division.

Second, insert transition frequency division between 7 frequency division and rated frequency of power grid, such as 4, 3 and 2 frequency division.

Third, it is a relatively large span from 2 to rated frequency of power grid. The ideal speed of the motor running at 2 frequency division is only half of the rated speed, so it can' $t$ be switched directly to power frequency. Otherwise, the slip s is too large to produce a large impact current. In order to avoid this situation, it is necessary to control the voltage. In this paper, the ramp soft starting mode is adopted. To obtain the maximum forward torque under the non-positive sequence, the symmetrical component method is used to analyze the phase of 3 and 2 frequency division, as shown in the Eq.(4)

$$
\left[\begin{array}{ccc}
\dot{U}_{n a}^{0} & \dot{U}_{n b}^{0} & \dot{U}_{n c}^{0} \\
\dot{U}_{n a}^{+} & \dot{U}_{n b}^{+} & \dot{U}_{n c}^{+} \\
\dot{U}_{n a}^{-} & \dot{U}_{n b}^{-} & \dot{U}_{n c}^{-}
\end{array}\right]=\frac{1}{3}\left[\begin{array}{ccc}
1 & 1 & 1 \\
1 & \alpha & \alpha^{2} \\
1 & \alpha^{2} & \alpha
\end{array}\right]\left[\begin{array}{lll}
\dot{U}_{n a} & \dot{U}_{n b} & \dot{U}_{n c} \\
\dot{U}_{n b} & \dot{U}_{n c} & \dot{U}_{n a} \\
\dot{U}_{n c} & \dot{U}_{n a} & \dot{U}_{n b}
\end{array}\right]
$$

The results show that the phase $\left(0^{\circ}, 260,100^{\circ}\right),\left(0^{\circ}, 260^{\circ}, 160^{\circ}\right)$ and $\left(0^{\circ}, 200^{\circ}, 100^{\circ}\right)$ have the maximum positive sequence component and the minimum negative sequence component in $16.7 \mathrm{~Hz}$, and the phase $\left(0^{\circ}, 210^{\circ}, 60^{\circ}\right),\left(0^{\circ}, 210^{\circ}, 150^{\circ}\right)$ and $\left(0^{\circ}, 300^{\circ}, 150^{\circ}\right)$ have the same contribution in $25 \mathrm{~Hz}$.

Based on the above analysis, the whole process of discrete frequency soft start is $\mathrm{f} / 13->\mathrm{f} / 7-\mathrm{f} / 4-\mathrm{f} / 3-\mathrm{f} / 2-$ ramp soft start-f. The following principles should be followed when switching frequency: 1) to ensure the continuity of the sine curve in the process of soft start; 2) to ensure the alternation of the positive and negative half cycle of the selected frequency; 3) to ensure the smooth transition of the torque before or after switching frequency, in order to reduce the torque oscillation. On the basis of these principles, the most simple and effective method is to switch frequency at the 
whole cycle or half cycle.

\section{Control Strategy of Trigger Angle}

The most important performance parameters of the motor starting process are the starting torque ratio and the starting current ratio, which require to obtain a larger starting torque with the minimum starting current. According to the asynchronous motor theory and its $\mathrm{T}$ type equivalent circuit, as shown in Fig.2, the starting torque $T_{s t}$ is given by

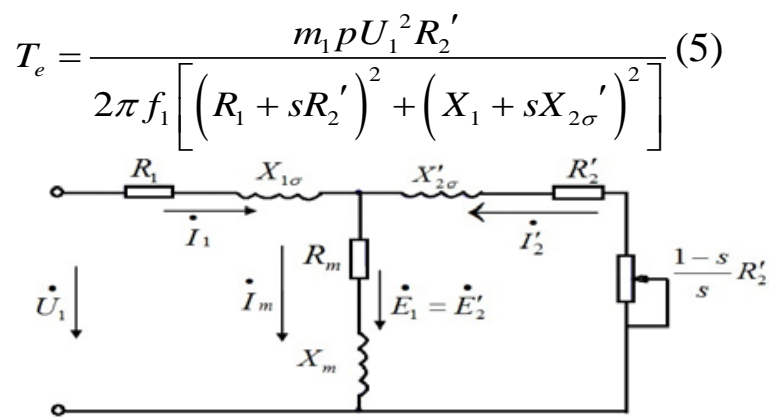

Fig. 2 T-type equivalent circuit of asynchronous motor

When the motor is starting, speed $n=0$ and slip $s=1$, starting torque and starting current is

$$
\begin{aligned}
& T_{s t}=\frac{m_{1} p}{2 \pi f_{1}} \frac{U_{1}^{2} R_{2}^{\prime}}{\left[\left(R_{1}+R_{2}^{\prime}\right)^{2}+\left(X_{1 \sigma}+X_{2 \sigma}^{\prime}\right)^{2}\right]} \\
& I_{s t} \approx \frac{U_{1}}{\sqrt{\left(R_{1}+R_{2}\right)^{2}+\left(X_{1 \sigma}+X_{2 \sigma}^{\prime}\right)^{2}}}
\end{aligned}
$$

The formula shows that the starting torque $T_{\text {st }}$ is proportional to the square of the input voltage, the starting current $I_{s t}$ is proportional to the input voltage, and impedance $X_{1}$ and $X_{2 \sigma}^{\prime}$ is proportional to the frequency of the power supply. In the discrete frequency soft start process, we hope to maintain the rated flux invariantly With frequency decreased, the input voltage of the motor is reduced by adjusting the trigger angle. This method can not only ensure the flux stability, but also maintain the basic torque constant, so as to achieve high torque. This is the basic principle of VVVF motor speed regulation, as in [6]. How to control the trigger angle? A simple method is to keep the trigger angle fixed in a certain frequency band. But this method has such harmonic content, that the motor jitter very seriously.Equivalent sine control method, deriving from the area equivalence principle, is adopted in this paper. That is to change the trigger angle rather than fixed in this certain frequency band to make output voltage close to sine wave, so as to reduce the impact of harmonics and torque ripple on the motor.

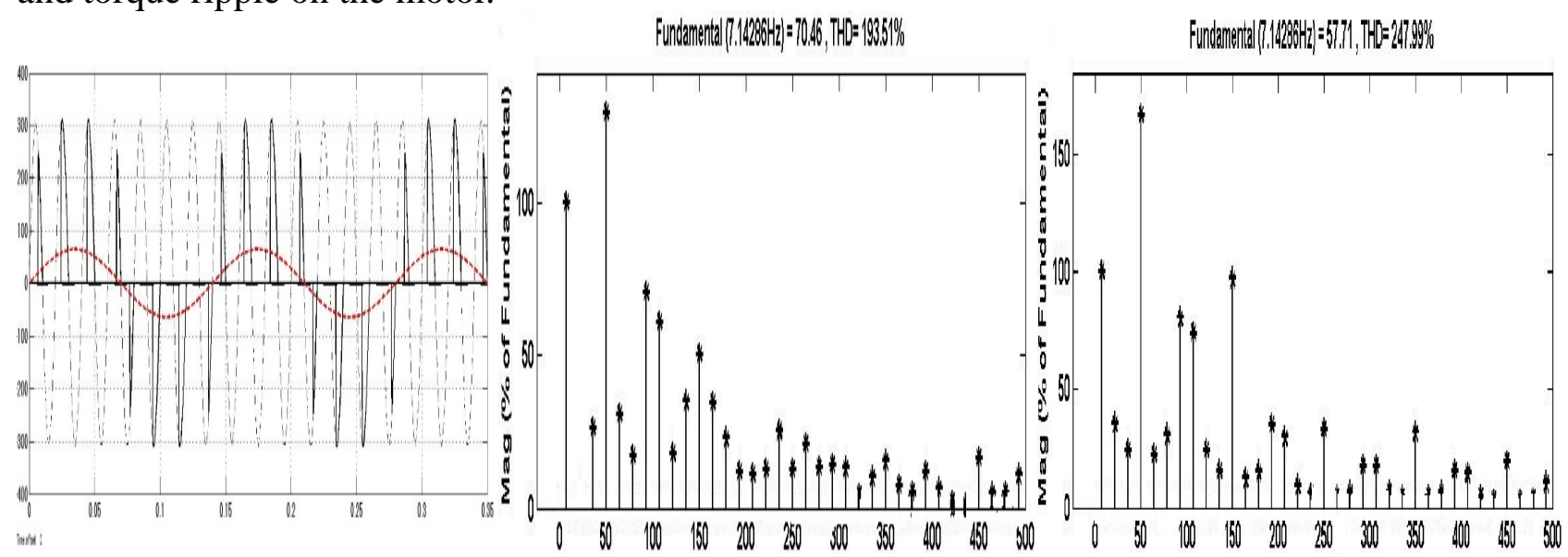

Fig. 3. Voltage output wave diagram of 7 hierarchical frequency

Fig. 4 Voltage output harmonic wave analysis of equivalent sine control of 7 discrete frequency Fig. 5 Voltage output harmonic wave analysis of fixed firing angle of 7 discrete frequency 
Fig. 3 is the output voltage wave diagram of 7 hierarchical frequency, where the solid line is fundamental voltage of seven frequency division $u_{v}=U_{v m} \sin \omega_{v} t$, and the dotted line is power frequency voltage $u_{1}=U_{1 m} \sin \omega_{1} t$. According to the principle of VVVF and equivalent sine control, trigger angle calculation formula is given by

$$
\left\{\begin{array}{l}
\int_{x_{k}}^{0.01} U_{1 m} \sin \omega_{1} t d t=\int_{2(k-1) \pi}^{2 k \pi} U_{v m} \sin \omega_{v} t d t \quad k=1,2,3 \\
\int_{x_{k}}^{0.01} U_{1 m} \sin \omega_{1} t d t=\int_{2(k-1) \pi}^{7 \pi} U_{v m} \sin \omega_{v} t d t k=4
\end{array}\right.
$$

where $U_{v m}=\tau U_{1 m} / v$ and $\tau$ is voltage compensation coefficient as low frequency.After getting the trigger time $x_{k}$, we can convert $x_{k}$ to the corresponding trigger angle through the formula (9)

$$
\alpha_{k}=\frac{180 x_{k}}{0.01} \quad k=1,2,3,4
$$

In order to show the advantages of equivalent sine control, I establish FFT simulation models in Simulink. Fig.3 for the equivalent sine trigger mode and Fig. 4 for fixed angle trigger mode, their fundamental frequencies are all $7.143 \mathrm{~Hz}$.From the image above we can learn that the THD is reduced to $193.51 \%$ after the equivalent sine trigger mode is adopted. However, the THD of the fixed angle trigger mode is $247.99 \%$. It can be known that this method optimized the voltage output waveform and, to a certain extent, improve the motor starting performance.

\section{Simulation Analysis}

With the aid of MATLAB/Simulink simulation platform (e.g. [7], [8]), I build the simulation system as shown in Fig.6. The asynchronous motor's power is $2.2 \mathrm{~kW}$, line voltage is $380 \mathrm{~V}$; stator and rotor resistance p.u.is respectively 3.478 and 2.546; stator, rotor and mutual inductance p.u.is respectively $12.54 \mathrm{mH}$ and $12.26 \mathrm{mH}, 332.9 \mathrm{mH}$; GD is $0.02 \mathrm{~kg} \cdot \mathrm{m}^{2}$; pole pairs are 2 . Starting strategy adopted in this paper is f/13->f/7-f/4-f/3-f/2-ramp soft start-f shown in Fig.7.

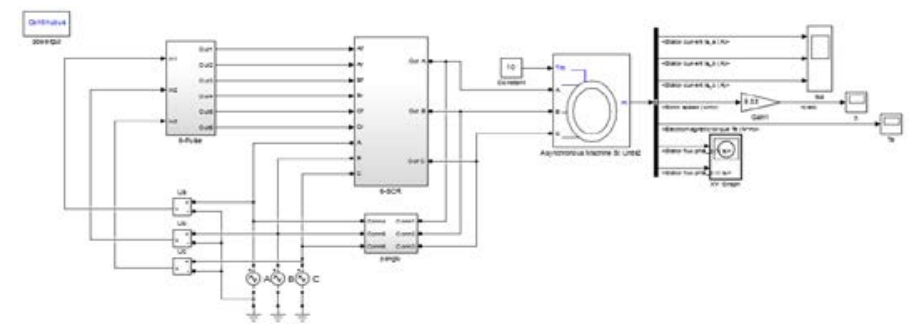

Fig. 6 Simulation System Diagram

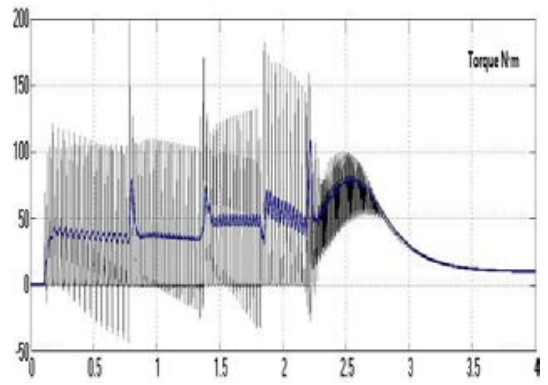

a) Torque simulation waveform

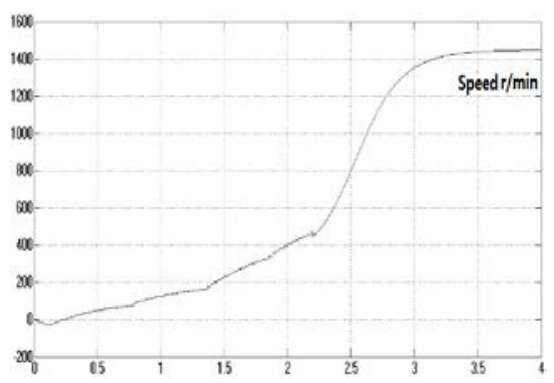

b)Speed simulation waveform

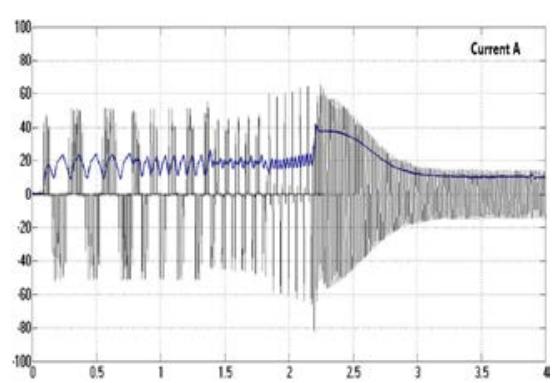

c) Current simulation waveform

Fig. 7 The simulation waveforms are under discrete frequency soft start 


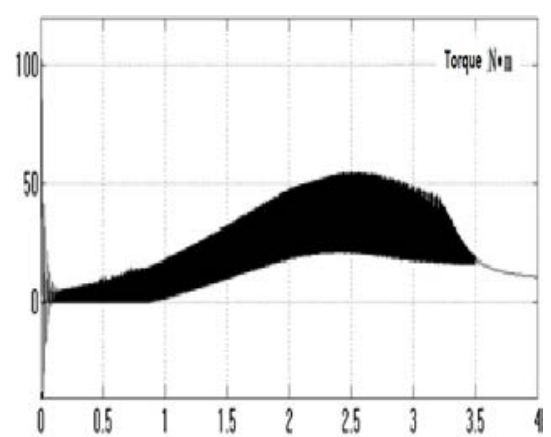

a) Torque simulation waveform

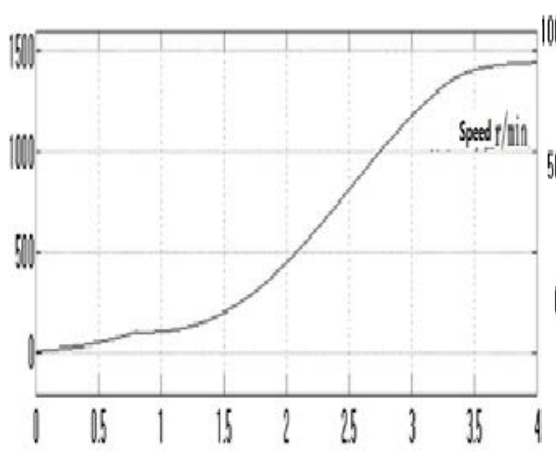

b) Speed simulation waveform

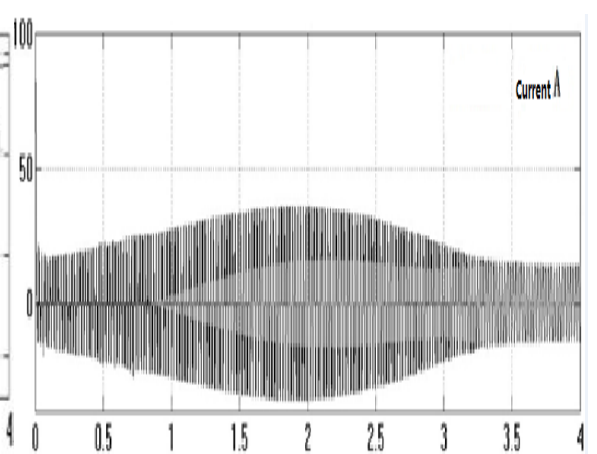

c) Current simulation waveform

Fig. 8 The simulation waveforms are under ramp voltage soft starter

Compared with the ramp voltage soft starter (as shown in Fig.8, the starting current of discrete frequency soft starter (as shown in Fig.7c) )is smaller than ramp voltage soft starter(as shown in Fig.8c) under the same load condition. And compared with the starting torque of the ramp voltage soft starter(as shown in Fig. 8a), the starting torque of discrete frequency soft can obtain a larger starting one( as shown in Fig.7)), and a relatively stable speed rise (as shown in Fig.7b). So the simulation experiment prove the rationality and validity of the application of the discrete frequency in the motor soft start.

\section{Prototype Design and Experiment}

\subsection{Control System Design}

Controller used in prototype design is a 16 bit high performance MCU (dsPIC6014A-301/PF) in Microchip Ltd., and main circuit is same with the traditional soft starter. Control circuit mainly includes: the synchronous voltage and phase sequence detection circuit, power supply circuit, driving circuit, current detection circuit,a contactor control circuit, keys and display. Soft start control system block diagram shown in Fig. 9.

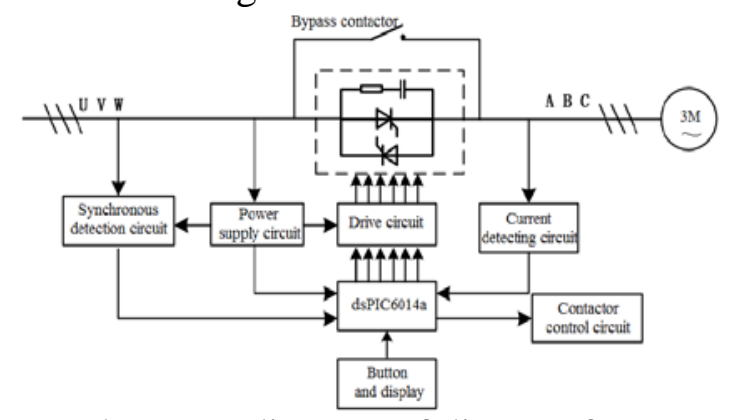

Fig. 9 The control system diagram of discrete frequency soft starter

\subsection{Voltage Synchronization and Trigger Scheme}

The trigger pulse of the main circuit for the six channel is required to maintain a strict phase relationship with the power supply voltage, and it is a key step in the design. Because thermistors is not only to realize the phase controlled voltage regulation, but to determine whether the corresponding half wave is triggered, and, what's more, to distribute the trigger angle according to the trigger rules of discrete frequency and the equivalent sine principle. The pulse trigger unit is shown in Fig.10. 


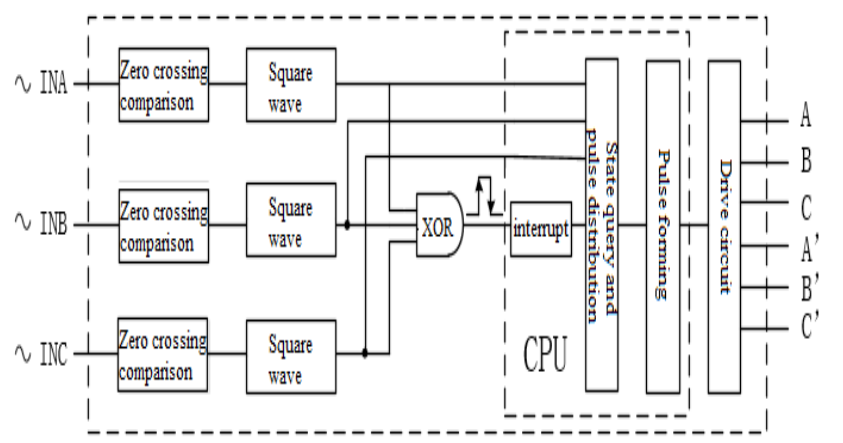

Fig. 10 The diagram of synchronization and trigger pulse

\subsection{Experimental Result}

The experiment object is a squirrel cage induction motor with rated voltage $380 \mathrm{~V} / 660 \mathrm{~V}$, rated current of $11 \mathrm{~A}$, rated power $5.5 \mathrm{KW}$, rated speed $2900 \mathrm{r} / \mathrm{min}$ and fan load. I will do tests on discrete frequency soft-starting and ramp voltage soft-starting. The discrete frequency numbers adopted in this experiment are 13, 7, 4, 3, 2, and plus ramp voltage soft-start and power frequency. Thus the seven- stage starting process is completed.

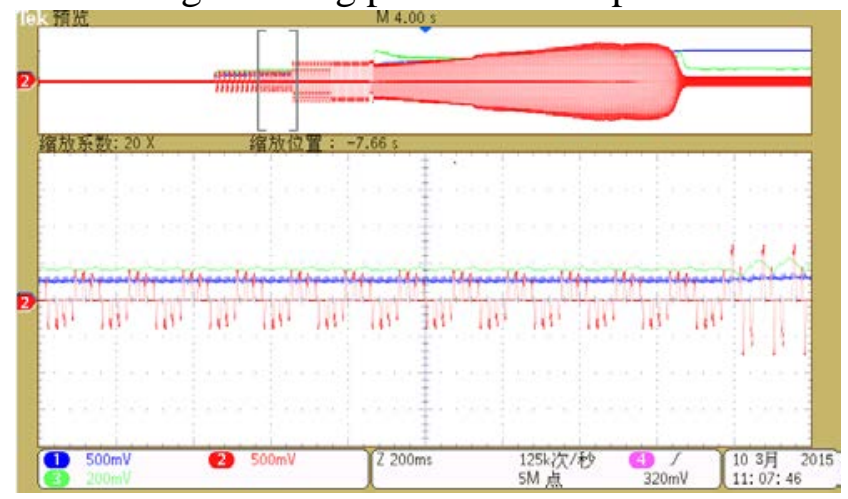

a) The waveform of discrete frequency soft start

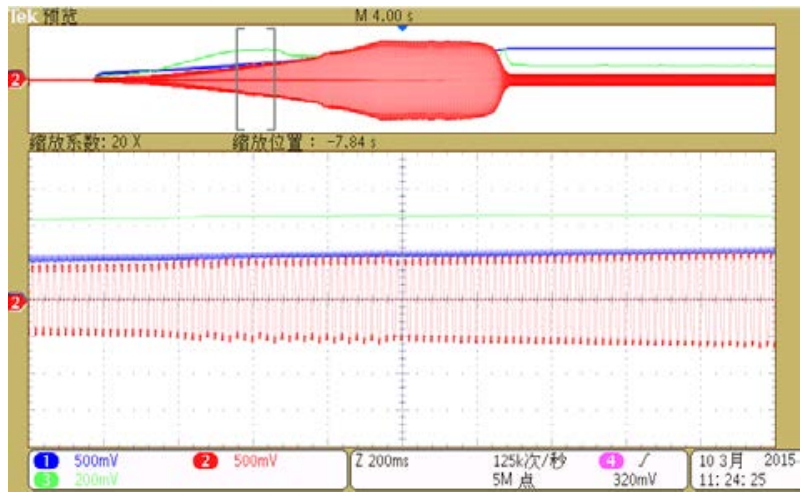

b) The waveform of ramp soft starter

Fig. 11 Experimental waveforms

The discrete frequency soft starter and ramp voltage soft starter's current waveform are shown in Fig. 11. The channel one means effective value of stator's phase voltage (50V each grid in ordinate). The channel two represents starting current (20A each grid in ordinate). The channel three signifies effective value of starting current (each grid is $200 \mathrm{~ms}$ in abscissa and 10A each grid in ordinate).

According to the comparison of experiments, it shows that the control effect of discrete frequency soft-starter is good and stable. The most successful thing is starting current is small and the current waveform complying with sine equivalence principle show approximately sine waveform. The chart 11a is partial enlarged view of 7 frequency current waveform.

\section{Conclusions}

Because starting torque is limited, the application of the traditional soft starter has been refrained. By analyzing the basic theory of discrete frequency and combining control strategy of equivalent sine, this thesis's results achieved high starting torque of the motor. The simulation and related experiment verified the correctness and validity of the theory. Without changing the original structure of the soft-starter, changing the control strategy can achieve discrete frequency soft-starting. This method make the output torque of the motor larger, meanwhile, decrease the starting current and meet the stable starting needs under the overload condition. Boasting starting performance improvement, broadening soft-starter application, this method enjoys high practical value. 


\section{References}

[1] YAN Yao, WANG Honghua. Development of Discrete Variable Frequency Induction Motors Soft Starter[A]. Machine Building \& Automation, 2012, 41(6):196-199.

[2] ZHAO Kai-qi, WANG Yi, XU Dianguo. A New Strategy To Improve Electromagnetic Starting Torque For Thyristor Controlled Induction Motors [J]. Proceedings of the CSEE, 2004, 24 (3) :145-150.

[3] XUN Pu-rong. Principle and Implementation of High Torque Soft-starter Adapted Discrete Cocloconvert [J]. Principle and Implementation, 2005, (02):1-5.

[4] WANG Zhao-an, LIU Jinjun. Power electronics technology [M]. Beijing: Mechanical Industry Press, 2009. 140-184.

[5] HU Hong-ming, MAO Chen-xiong, LU Ji-ming, YUAN You-xin. Discrete Frequency Principle and Simulation of High Torque Soft Starter [A]. Electric Drive, 2009, 39(2) :16-19.

[6] YANG Shun-chang, LIAO Yong, YU Yu. Analysis and Calcualation of Electromagnetic Torques for AC Excited Motors Incorporating the Effect of Harmonic Currents in the Excitation Source [J]. Transactions of China Electrotechnical Society, 2003, 18(1): 5-9.

[7] SUN Jin-ji, FANG Jian-cheng, WANG Jian-min. Oscillation in Soft-Starting of Induction Motor[J]. Transactions of China Electrotechnical Society, 2007, 22(2): 15-21

[8] TONG Jun, ZHANG Zhen, GUO Chang-Yong. Study of Power Factor Angle closed-loop Control Technology in soft-starter[A]. Electric Machines and Control, 2013, 17 (12): 51-56. 